\title{
BASS NUMBERS OF LOCAL COHOMOLOGY MODULES
}

\author{
CRAIG L. HUNEKE AND RODNEY Y. SHARP
}

\begin{abstract}
Let $A$ be a regular local ring of positive characteristic. This paper is concerned with the local cohomology modules of $A$ itself, but with respect to an arbitrary ideal of $A$. The results include that all the Bass numbers of all such local cohomology modules are finite, that each such local cohomology module has finite set of associated prime ideals, and that, whenever such a local cohomology module is Artinian, then it must be injective. (This last result had been proved earlier by Hartshorne and Speiser under the additional assumptions that $A$ is complete and contains its residue field which is perfect.) The paper ends with some low-dimensional evidence related to questions about whether the analogous statements for regular local rings of characteristic 0 are true.
\end{abstract}

\section{INTRODUCTION}

Let $A$ be a commutative Noetherian ring, let $\mathfrak{b}$ be an ideal of $A$ and let $M$ be a finitely generated $A$-module. Grothendieck [G2, Exposé XIII, 1.1] conjectured that the local cohomology modules $H_{\mathfrak{b}}^{j}(M)$ of $M$ with respect to $\mathfrak{b}$ are such that $\operatorname{Hom}_{A}\left(A / \mathfrak{b}, H_{\mathfrak{b}}^{j}(M)\right)$ is finitely generated for all $j \in \mathbb{N}_{0}$. (We use $\mathbb{N}_{0}$ (respectively $\mathbb{N}$ ) to denote the set of nonnegative (respectively positive) integers.) Hartshorne [H2, §3] showed that Grothendieck's conjecture is false in the stated generality, but went on to investigate the conjecture in the special case in which $A$ is a complete regular local ring: he showed that, in this case, $\operatorname{Ext}_{A}^{i}\left(A / \mathfrak{b}, H_{\mathfrak{b}}^{j}(M)\right)$ is finitely generated for all $i, j \in \mathbb{N}_{0}$ if either $\mathfrak{b}$ is a nonzero principal ideal $[\mathrm{H} 2,6.3]$ or $\mathfrak{b}$ is a prime ideal with $\operatorname{dim} A / \mathfrak{b}=1[\mathrm{H} 2$, 7.7].

Hartshorne's work provides motivation for study of the corresponding questions in the special case in which $M=A$ (and $A$ is still a regular local ring). Recently, the present first author and Koh obtained the intriguing result $[\mathrm{H}$ $\mathrm{K}, 2.3]$ that if $A$ is a regular local ring of characteristic $p>0$ and $j \in \mathbb{N}$ is such that $j>\operatorname{bight}(\mathfrak{b})$ and $\operatorname{Hom}_{A}\left(A / \mathfrak{b}, H_{\mathfrak{b}}^{j}(A)\right)$ is finitely generated, then $H_{\mathfrak{b}}^{j}(A)=0$; here, bight $(\mathfrak{b})$, the bigheight of $\mathfrak{b}$, is defined by

$$
\operatorname{bight}(\mathfrak{b})=\max \{h t \mathfrak{p}: \mathfrak{p} \text { is a minimal prime ideal of } \mathfrak{b}\} .
$$

Received by the editors July 12, 1991.

1980 Mathematics Subject Classification (1985 Revision). Primary 13H05, 14B15, $13 \mathrm{C} 11$.

Key words and phrases. Regular local ring, characteristic $p$, Frobenius homomorphism, local cohomology module, Bass number, associated prime ideal, Artinian module, injective module.

The first author was supported in part by the NSF and in part by the United Kingdom SERC (Grant number GR/F71836). 
The aim of the present paper is to obtain more information about the structure of the local cohomology modules $H_{\mathfrak{b}}^{j}(A)\left(j \in \mathbb{N}_{0}\right)$, where $\mathfrak{b}$ is an arbitrary ideal of the regular local ring $A$ of characteristic $p>0$. We make much use of the Frobenius homomorphism $f: A \rightarrow A$ defined by $f(a)=a^{p}$ for all $a \in A$. Peskine and Szpiro [P-S] pioneered the use of the Frobenius homomorphism in commutative algebra over commutative Noetherian rings of prime characteristic, and one could take the view that this paper merely pushes their arguments a little further. We shall prove that, for all $i, j \in \mathbb{N}_{0}$ and for all $\mathfrak{p} \in \operatorname{Spec}(A)$, the Bass number $\mu^{i}\left(\mathfrak{p}, H_{\mathfrak{b}}^{j}(A)\right)$ is finite (and bounded above by $\left.\mu^{i}\left(\mathfrak{p}, \operatorname{Ext}_{A}^{j}(A / \mathfrak{b}, A)\right)\right)$; a corollary is that $\operatorname{Ass}\left(H_{\mathfrak{b}}^{j}(A)\right)$ is finite (for all $\left.j \in \mathbb{N}_{0}\right)$.

In a slightly different direction, we shall also present some results about the structure of $H_{\mathrm{b}}^{j}(A)$ in the case when this local cohomology module is Artinian (that is, in the terminology of Hartshorne [H2, $\S 1]$, $\mathfrak{m}$-cofinite, where $\mathfrak{m}$ denotes the maximal ideal of $A$ ). Hartshorne and Speiser [H-S, 2.4] proved, again in the situation of the preceding paragraph but with the additional assumptions that $A$ is complete and contains its residue field which is perfect, that if $H_{\mathfrak{b}}^{j}(A)$ is Artinian, then it is injective and isomorphic to a direct sum of finitely many copies of $E_{A}(A / \mathrm{m})$ (we use $E_{A}(L)$ or $E(L)$ to denote the injective envelope of an $A$-module $L$ ). We shall obtain the same result for a general regular local ring $(A, \mathfrak{m})$ of characteristic $p>0$, without any restriction on the residue field. In fact, our argument yields rather more than this: we shall also show that, for all $i, j \in \mathbb{N}_{0}$, the local cohomology module $H_{\mathfrak{m}}^{i}\left(H_{\mathfrak{b}}^{j}(A)\right)$ is always injective, and isomorphic to the direct sum of finitely many copies of $E(A / \mathfrak{m})$.

The above-mentioned results raise obvious questions about whether the analogous statements for regular local rings of characteristic 0 are true. In the final section of the paper, we include some low-dimensional evidence related to such questions, including proofs that, if $(A, \mathfrak{m})$ is a regular local ring of characteristic 0 having $\operatorname{dim} A \leqslant 3$, or a 4-dimensional regular local ring containing a field of characteristic 0 , and $\mathfrak{b}$ is an ideal of $A$, then the Bass number $\mu^{i}\left(\mathfrak{p}, H_{\mathfrak{b}}^{j}(A)\right)$ is finite for all $i, j \in \mathbb{N}_{0}$ and all $\mathfrak{p} \in \operatorname{Spec}(A)$.

\section{Preliminaries}

Throughout the paper, $A$ will denote a commutative Noetherian ring. We shall only assume that $A$ is local, or of characteristic $p>0$, when explicitly stated; however, the terminology ' $(A, \mathfrak{m})$ is a local ring' is to be interpreted as including the information that $\mathfrak{m}$ is the unique maximal ideal of $A$.

We assume that the reader is familiar with the basic construction and properties of local cohomology modules, as introduced in [G1].

The Bass numbers $\mu^{i}(\mathfrak{p}, M)\left(i \in \mathbb{N}_{0}\right)$ for an $A$-module $M$ and a prime ideal $\mathfrak{p}$ of $A$ are explained as follows. For $i \in \mathbb{N}_{0}$, the $i$ th term $E^{i}(M)$ in the minimal injective resolution for $M$ is uniquely determined, up to isomorphism, by $M$; by well-known work of Matlis and Gabriel, there is a family $\left(\mathfrak{p}_{\alpha}\right)_{\alpha \in \Lambda}$ of prime ideals of $A$ for which $E^{i}(M) \cong \bigoplus_{\alpha \in \Lambda} E\left(A / \mathfrak{p}_{\alpha}\right)$, and, for $\mathfrak{p} \in \operatorname{Spec}(A)$, the cardinality of the set $\left\{\alpha \in \Lambda: \mathfrak{p}_{\alpha}=\mathfrak{p}\right\}$ is uniquely determined by $M$ and is denoted by $\mu^{i}(\mathfrak{p}, M)$. In fact, by Bass [B, (2.7)], this can be described as the dimension of a vector space: if $k(\mathfrak{p})$ denotes the residue field of the local ring $A_{\mathfrak{p}}$, then 


$$
\mu^{i}(\mathfrak{p}, M)=\operatorname{dim}_{k(\mathfrak{p})} \operatorname{Ext}_{A_{\mathfrak{p}}}^{i}\left(k(\mathfrak{p}), M_{\mathfrak{p}}\right)=\operatorname{dim}_{k(\mathfrak{p})}\left(\operatorname{Ext}_{A}^{i}(A / \mathfrak{p}, M)\right)_{\mathfrak{p}},
$$

and so is finite when $M$ is finitely generated.

1.1 Notation. Let $A$ have prime characteristic $p>0$. We use $f: A \rightarrow A$ to denote the Frobenius homomorphism; we shall occasionally use $A^{\prime}$ to denote $A$ regarded as an $A$-module by means of $f$ (at points where care is needed). Also, $F$ will denote the additive functor $(\quad) \otimes_{A} A^{\prime}$ from the category of all $A$-modules and $A$-homomorphisms to the category of all $A^{\prime}$-modules and $A^{\prime}$ homomorphisms, and we shall refer to $F$ as the Frobenius functor.

For an ideal $c$ of $A$, and for $e \in \mathbb{N}$, we shall denote by $c^{\left[p^{e}\right]}$ the ideal of $A$ generated by all the $p^{e}$ th powers of elements of $\mathfrak{c}$. Note that $\left(\mathfrak{c}^{\left[p^{e}\right]}\right)^{[p]}=\mathfrak{c}^{\left[p^{e+1}\right]}$.

1.2 Remark. Let the situation be as in 1.1. For an ideal c of $A$, we have $F(A / \mathfrak{c}) \cong A / \mathfrak{c}^{[p]}$.

1.3 Remark. Let $(A, \mathfrak{m})$ be a regular local ring of characteristic $p>0$.

Our argument is very dependent on Kunz's Theorem [K] that, in this case, the Frobenius homomorphism $f$ is flat. For example, we shall need the consequence that

$$
F\left(\mathrm{Ext}_{A}^{j}(M, N)\right) \cong \mathrm{Ext}_{A^{\prime}}^{j}(F(M), F(N))
$$

for all $j \in \mathbb{N}_{0}$ and for all choices of $A$-modules $M$ and $N$ with $M$ finitely generated.

Crucial to the arguments of this paper is the fact that, if $I$ is an injective module over a Gorenstein ring $A$ of prime characteristic $p>0$, then $F(I) \cong I$. The next lemma is in preparation for this.

1.4 Lemma. Let $(A, \mathfrak{m})$ be a Gorenstein local ring of prime characteristic $p>$ 0 , and let $E:=E(A / \mathfrak{m})$. Then $F(E) \cong E$.

Proof. Let $n:=\operatorname{dim} A$, and let $x_{1}, \ldots, x_{n}$ form a system of parameters for $A$. Since $A$ is Gorenstein, $E \cong H_{\mathfrak{m}}^{n}(A)$. We are going to use the description of this local cohomology module as a direct limit of homology modules of Koszul complexes: see Grothendieck [G1, Theorem 2.3].

For $r, s \in \mathbb{N}$ with $r \leqslant s$, there is an $A$-homomorphism

$$
g_{r}^{s}: A /\left(x_{1}^{r}, \ldots, x_{n}^{r}\right) \rightarrow A /\left(x_{1}^{s}, \ldots, x_{n}^{s}\right)
$$

induced by multiplication by $\left(x_{1}, \ldots, x_{n}\right)^{s-r}$; these homomorphisms turn

$$
\left(A /\left(x_{1}^{r}, \ldots, x_{n}^{r}\right)\right)_{r \in \mathbb{N}}
$$

into a direct system, and it follows from the result cited in the preceding paragraph that

$$
H_{\mathrm{m}}^{n}(A)=H_{\left(x_{1}, \ldots, x_{n}\right)}^{n}(A) \cong \underset{r \in \mathrm{N}}{\lim _{\longrightarrow}} A /\left(x_{1}^{r}, \ldots, x_{n}^{r}\right) .
$$

Now apply the functor $F$, and use 1.2 and the fact that the formation of tensor products and direct limits 'commute': it is easy to deduce that

$$
F(E) \cong F\left(\lim _{r \in \mathbb{N}} A /\left(x_{1}^{r}, \ldots, x_{n}^{r}\right)\right) \cong H_{\left(x_{1}^{p}, \ldots, x_{n}^{p}\right)}^{n}(A)=H_{\mathfrak{m}}^{n}(A) \cong E .
$$


1.5 Proposition. Let $A$ be a Gorenstein ring of prime characteristic $p>0$, and let $I$ be an injective A-module. Then $F(I) \cong I$.

Proof. Let $\mathfrak{p} \in \operatorname{Spec}(A)$ : we show first that $F(E(A / \mathfrak{p})) \cong E(A / \mathfrak{p})$.

Now, by Matlis [M1, Lemma (3.2)(2) and Theorem (3.4)], each element of $E(A / \mathfrak{p})$ is annihilated by some power of $\mathfrak{p}$, and multiplication by an element of $A \backslash \mathfrak{p}$ provides an automorphism of $E(A / \mathfrak{p})$. Hence $E(A / \mathfrak{p})$ has a natural structure as an $A_{\mathfrak{p}}$-module; in fact, as such, it is isomorphic to $E_{A_{\mathfrak{p}}}\left(A_{\mathfrak{p}} / \mathfrak{p} A_{\mathfrak{p}}\right)$.

It follows that each element of $F(E(A / \mathfrak{p}))$ is annihilated by some power of $\mathfrak{p}$, and multiplication by an element of $A \backslash \mathfrak{p}$ provides an automorphism of $F(E(A / \mathfrak{p}))$. Hence $F(E(A / \mathfrak{p}))$ has a natural structure as an $A_{\mathfrak{p}}$-module, and

$$
F(E(A / \mathfrak{p})) \otimes_{A} A_{\mathfrak{p}} \cong F(E(A / \mathfrak{p}))
$$

as $A_{\mathfrak{p}}$-modules.

Note that $A_{\mathfrak{p}}$ is a Gorenstein local ring of characteristic $p:$ let $f^{\prime}: A_{\mathfrak{p}} \rightarrow$ $A_{\mathfrak{p}}$ denote the Frobenius homomorphism and let $F^{\prime}$ denote the Frobenius functor on the category of $A_{\mathfrak{p}}$-modules. Let $\sigma: A \rightarrow A_{\mathfrak{p}}$ denote the natural homomorphism. Note that the diagram

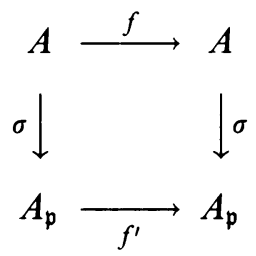

commutes. Hence there is an $A_{\mathfrak{p}}$-isomorphism

$$
F(E(A / \mathfrak{p})) \otimes_{A} A_{\mathfrak{p}} \cong F^{\prime}\left(E(A / \mathfrak{p}) \otimes_{A} A_{\mathfrak{p}}\right),
$$

as both of these $A_{\mathfrak{p}}$-modules are isomorphic to the $A_{\mathfrak{p}}$-module $E(A / \mathfrak{p}) \otimes_{A} A_{\mathfrak{p}}$ which arises when $A_{\mathfrak{p}}$ is regarded as an $A$-module by means of $\sigma \circ f=f^{\prime} \circ \sigma$.

But $E(A / \mathfrak{p}) \otimes_{A} A_{\mathfrak{p}} \cong E_{A_{\mathfrak{p}}}\left(A_{\mathfrak{p}} / \mathfrak{p} A_{\mathfrak{p}}\right)$, and it follows from 1.4 that

$$
F^{\prime}\left(E_{A_{\mathfrak{p}}}\left(A_{\mathfrak{p}} / \mathfrak{p} A_{\mathfrak{p}}\right)\right) \cong E_{A_{\mathfrak{p}}}\left(A_{\mathfrak{p}} / \mathfrak{p} A_{\mathfrak{p}}\right)
$$

as $A_{\mathfrak{p}}$-modules. Hence there are isomorphisms of $A_{\mathfrak{p}}$-modules

$$
F(E(A / \mathfrak{p})) \cong F(E(A / \mathfrak{p})) \otimes_{A} A_{\mathfrak{p}} \cong F^{\prime}\left(E_{A_{\mathfrak{p}}}\left(A_{\mathfrak{p}} / \mathfrak{p} A_{\mathfrak{p}}\right)\right) \cong E_{A_{\mathfrak{p}}}\left(A_{\mathfrak{p}} / \mathfrak{p} A_{\mathfrak{p}}\right),
$$

so that, on restricting back to $A$, we obtain an $A$-isomorphism

$$
F(E(A / \mathfrak{p})) \cong E(A / \mathfrak{p}) \text {. }
$$

Finally, we can deduce that $F(I) \cong I$ because $I$ is isomorphic to a direct sum of indecomposable injective $A$-modules (and tensor product 'commutes' with arbitrary direct sums).

1.6 Corollary. Let $(A, \mathfrak{m})$ be a regular local ring of characteristic $p>0$, and let $M$ be an A-module. Then $\operatorname{Ass}(F(M))=\operatorname{Ass}(M)$.

Proof. Note that we do not assume that $M$ is finitely generated. Let $E:=$ $E(M)$. By Kunz's Theorem [K], $F$ is exact, and so $F(M)$ can be embedded in $F(E)$, and $F(E) \cong E$ by 1.5 . Hence $E(F(M))$ is isomorphic to a direct summand of $E$. Also, for $\mathfrak{p} \in \operatorname{Spec}(A)$, we have $\mathfrak{p} \in \operatorname{Ass}(M)$ if and only if $\mu^{0}(\mathfrak{p}, M)>0$. It follows that $\operatorname{Ass}(F(M)) \subseteq \operatorname{Ass}(M)$. 
To prove the reverse inclusion, let $q \in \operatorname{Ass}(M)$, so that there is an exact sequence $0 \rightarrow A / \mathfrak{q} \rightarrow M$; application of the exact functor $F$ and 1.2 now show that $F(M)$ has a submodule isomorphic to $A / \mathfrak{q}^{[p]} ;$ and $\mathfrak{q} \in \operatorname{Ass}\left(A / q^{[p]}\right)$ since $\mathfrak{q}$ is the unique minimal prime ideal of $\mathfrak{q}^{[p]}$.

1.7 Remark. Let $(A, \mathfrak{m})$ be a local ring. It is straightforward to show that if $\left(L_{i}\right)_{i \in \mathbb{N}}$ is a direct system of $A$-modules (over the directed set $\mathbb{N}$ with its usual ordering) with direct limit $L_{\infty}$, and there exists $h \in \mathbb{N}$ such that the vector space dimensions (over $A / \mathfrak{m})$ of the socles $\left(0: L_{i} \mathfrak{m}\right)(i \in \mathbb{N})$ satisfy

$$
\operatorname{dim}_{A / \mathfrak{m}}\left(0:_{L_{i}} \mathfrak{m}\right) \leq h \text { for all } i \in \mathbb{N},
$$

then $\operatorname{dim}_{A / \mathfrak{m}}\left(0:_{L_{\infty}} \mathfrak{m}\right) \leq h$ also.

1.8 Lemma. Let $(A, \mathfrak{m})$ be a regular local ring of characteristic $p>0$, and let $\mathfrak{b}$ be an ideal of $A$. Let $j \in \mathbb{N}_{0}$.

Then $F\left(H_{\mathfrak{b}}^{j}(A)\right) \cong H_{\mathfrak{b}}^{j}(A)$.

Proof. Since the Frobenius homomorphism $f: A \rightarrow A$ is flat, and the extension of $\mathfrak{b}$ to $A$ under $f$ is just $\mathfrak{b}^{[p]}$, it follows from the theory of flat base change in local cohomology that $F\left(H_{\mathfrak{b}}^{j}(A)\right) \cong H_{\mathfrak{b}[p]}^{j}(A)$; but if $\mathfrak{b}$ can be generated by $t$ elements, then $\mathfrak{b}^{t p} \subseteq \mathfrak{b}^{[p]} \subseteq \mathfrak{b}^{p}$, and so $F\left(H_{\mathfrak{b}}^{j}(A)\right) \cong H_{\mathfrak{b}}^{j}(A)$.

\section{BASS NUMBERS}

2.1 Theorem. Let $(A, \mathfrak{m})$ be a regular local ring of characteristic $p>0$, and let $\mathfrak{b}$ be an ideal of $A$. Let $\mathfrak{p} \in \operatorname{Spec}(A)$, and let $j \in \mathbb{N}_{0}$. Then $\mu^{i}\left(\mathfrak{p}, H_{\mathfrak{b}}^{j}(A)\right)$ is finite for all $i \in \mathbb{N}_{0}$; in fact,

$$
\mu^{i}\left(\mathfrak{p}, H_{\mathfrak{b}}^{j}(A)\right) \leq \mu^{i}\left(\mathfrak{p}, \operatorname{Ext}_{A}^{j}(A / \mathfrak{b}, A)\right) \quad \text { for all } i \in \mathbb{N}_{0} .
$$

Proof. Localization enables us to reduce to the case in which $\mathfrak{p}=\mathfrak{m}$. For all $i \in \mathbb{N}_{0}$, set $h_{i}:=\mu^{i}\left(\mathfrak{m}, \operatorname{Ext}_{A}^{j}(A / \mathfrak{b}, A)\right)$.

Let

$$
0 \longrightarrow \operatorname{Ext}_{A}^{j}(A / \mathfrak{b}, A) \longrightarrow E^{0} \longrightarrow \cdots \longrightarrow E^{i} \longrightarrow E^{i+1} \longrightarrow \cdots
$$

provide a minimal injective resolution for $\operatorname{Ext}_{A}^{j}(A / \mathfrak{b}, A)$. For each $i \in \mathbb{N}_{0}$, set $E_{1}^{i}=F\left(E^{i}\right)$, and, for each $n \in \mathbb{N}$, define $E_{n+1}^{i}=F\left(E_{n}^{i}\right)$. Note that, by 1.5 , $E_{n}^{i} \cong E^{i}$ for all $n \in \mathbb{N}$ and all $i \in \mathbb{N}_{0}$.

Application of the exact functor $F$ to the above exact sequence yields, on use of 1.2 and 1.3 , an exact sequence

$$
0 \longrightarrow \operatorname{Ext}_{A}^{j}\left(A / b^{[p]}, A\right) \longrightarrow E_{1}^{0} \longrightarrow \cdots \longrightarrow E_{1}^{i} \longrightarrow E_{1}^{i+1} \longrightarrow \cdots
$$

But the natural homomorphism $A / \mathfrak{b}^{[p]} \rightarrow A / \mathfrak{b}$ (arising from the inclusion map $\left.\mathfrak{b}^{[p]} \rightarrow \mathfrak{b}\right)$ induces a natural homomorphism

$$
\operatorname{Ext}_{A}^{j}(A / \mathfrak{b}, A) \longrightarrow \operatorname{Ext}_{A}^{j}\left(A / \mathfrak{b}^{[p]}, A\right) .
$$

Standard results about injective resolutions of modules therefore mean that there is a commutative diagram

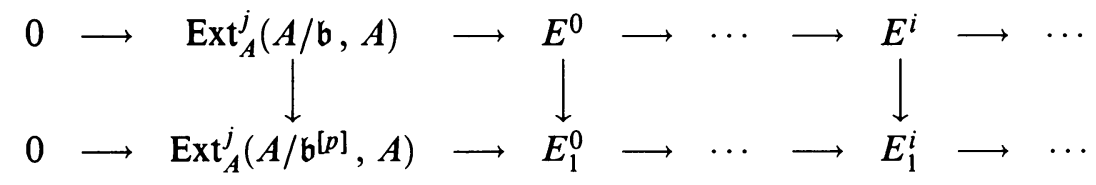


of $A$-modules and $A$-homomorphisms with exact rows, in which the first vertical map is the above-mentioned natural one. Indeed, repetition of this argument leads to a commutative diagram

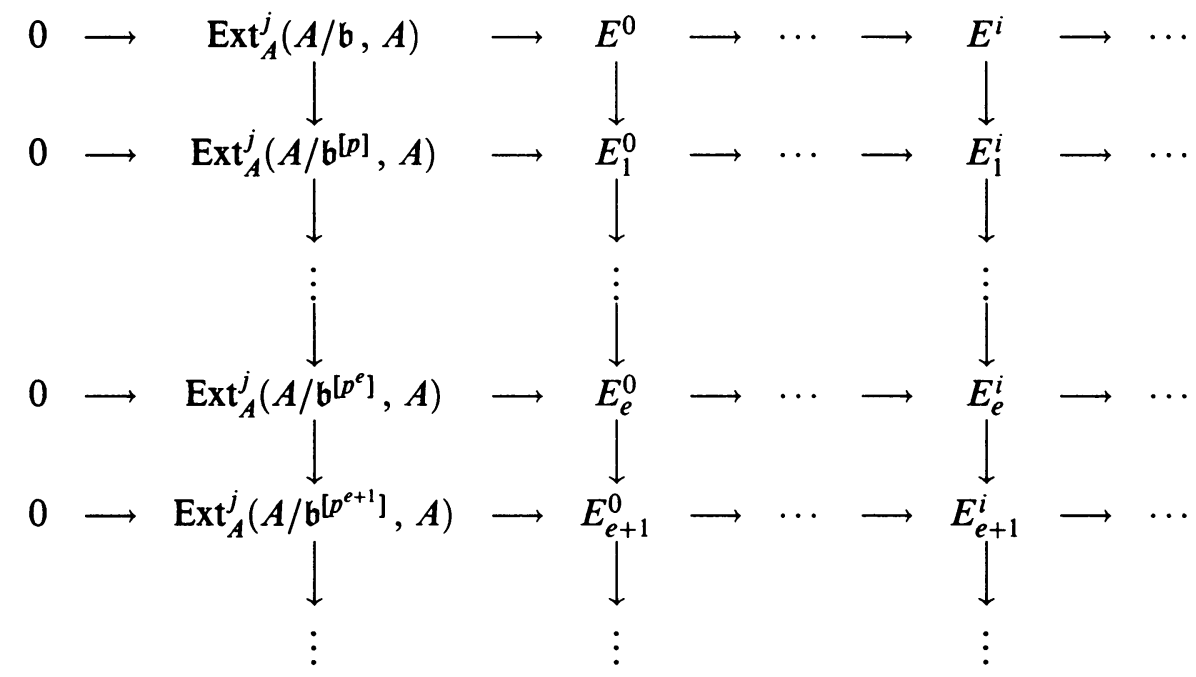

of $A$-modules and $A$-homomorphisms with exact rows, in which the maps in the first vertical column are all the natural ones.

Now pass to the direct limit: for each $i \in \mathbb{N}_{0}$, let

$$
J^{i}:=\underset{e \in \mathbb{N}}{\lim } E_{e}^{i},
$$

and recall that a direct limit of injective $A$-modules is again injective; recall also that passage to direct limits preserves exactness; and note too that, if $\mathfrak{b}$ can be generated by $t$ elements, then

$$
\mathfrak{b}^{t p^{e}} \subseteq \mathfrak{b}^{\left[p^{e}\right]} \subseteq \mathfrak{b}^{p^{e}} \quad \text { for all } e \in \mathbb{N},
$$

so that

$$
H_{\mathfrak{b}}^{j}(A) \cong \underset{e \in \mathbb{N}}{\lim _{e x}} \operatorname{Ext}_{A}^{j}\left(A / \mathfrak{b}^{\left[p^{e}\right]}, A\right) \quad \text { for all } e \in \mathbb{N} .
$$

The result is an exact sequence

$$
0 \longrightarrow H_{\mathfrak{b}}^{j}(A) \longrightarrow J^{0} \longrightarrow \cdots \longrightarrow J^{i} \longrightarrow J^{i+1} \longrightarrow \cdots
$$

which provides an injective resolution for $H_{\mathfrak{b}}^{j}(A)$. Now

$$
\operatorname{dim}_{A / \mathfrak{m}}\left(0:_{E_{e}^{i}} \mathfrak{m}\right)=h_{i} \quad \text { for all } e \in \mathbb{N} \text { and } i \in \mathbb{N}_{0},
$$

and so it follows from 1.7 that

$$
\operatorname{dim}_{A / \mathfrak{m}}\left(0:_{f^{i}} \mathfrak{m}\right) \leq h_{i} \quad \text { for all } i \in \mathbb{N}_{0} .
$$

Since, for each such $i$, the $i$ th term in the minimal injective resolution for $H_{\mathfrak{b}}^{j}(A)$ is isomorphic to a direct summand of $J^{i}$, it follows that $\mu^{i}\left(\mathfrak{m}, H_{\mathfrak{b}}^{j}(A)\right)$ is finite and no greater than $h_{i}$.

2.2 Remark. It is easy to produce examples of $(A, \mathfrak{m}), \mathfrak{b}, \mathfrak{p}, i$ and $j$ as in 2.1 for which

$$
\mu^{i}\left(\mathfrak{p}, H_{\mathfrak{b}}^{j}(A)\right)<\mu^{i}\left(\mathfrak{p}, \operatorname{Ext}_{A}^{j}(A / \mathfrak{b}, A)\right) .
$$


It might be interesting to try to determine conditions under which

$$
\mu^{i}\left(\mathfrak{p}, H_{\mathfrak{b}}^{j}(A)\right)=\mu^{i}\left(\mathfrak{p}, \operatorname{Ext}_{A}^{j}(A / \mathfrak{b}, A)\right)
$$

By a regular ring we mean a (commutative Noetherian) ring all of whose localizations are regular local rings.

2.3 Corollary. Let $A$ be a regular ring of positive characteristic, and let $\mathfrak{b}$ be an ideal of $A$. Let $j \in \mathbb{N}_{0}$. Then $\operatorname{Ass}\left(H_{\mathfrak{b}}^{j}(A)\right)$ is finite; in fact,

$$
\operatorname{Ass}\left(H_{\mathfrak{b}}^{j}(A)\right) \subseteq \operatorname{Ass}\left(\operatorname{Ext}_{A}^{j}(A / \mathfrak{b}, A)\right) .
$$

Proof. It is enough to prove the second statement, and to do that, we can assume that $A$ is a regular local ring of characteristic $p>0$. The claim is then immediate from 2.1 since, for $\mathfrak{p} \in \operatorname{Spec}(A)$ and an $A$-module $M$, we have $\mathfrak{p} \in \operatorname{Ass}(M)$ if and only if $\mu^{0}(\mathfrak{p}, M)>0$.

Our final result in this section gives a partial answer to a special case of a 'local-global' question raised by M. P. Brodmann.

2.4 Corollary. Let $A$ be a regular ring of positive characteristic, and let $\mathfrak{b}, \mathfrak{c}$ be ideals of $A$. Let $j \in \mathbb{N}_{0}$. Suppose that

$$
\mathfrak{c} A_{\mathfrak{p}} \subseteq \sqrt{ }\left(0:_{A_{\mathfrak{p}}} H_{\mathfrak{b} A_{\mathfrak{p}}}^{j}\left(A_{\mathfrak{p}}\right)\right) \text { for all } \mathfrak{p} \in \operatorname{Spec}(A) .
$$

Then $\mathfrak{c} \subseteq \sqrt{ }\left(0:{ }_{A} H_{\mathfrak{b}}^{j}(A)\right)$.

Proof. Clearly, we can assume that $H_{\mathfrak{b}}^{j}(A) \neq 0$. By 2.4, $\operatorname{Ass}\left(H_{\mathfrak{b}}^{j}(A)\right)$ is finite: let $\operatorname{Ass}\left(H_{\mathfrak{b}}^{j}(A)\right)=\left\{\mathfrak{p}_{1}, \ldots, \mathfrak{p}_{n}\right\}$. By hypothesis, for each $i=1, \ldots, n$, there exists $t_{i} \in \mathbb{N}$ such that

$$
\mathfrak{c}^{t_{i}} A_{\mathfrak{p}_{i}}\left(H_{\mathfrak{b}}^{j}(A)\right)_{\mathfrak{p}_{i}}=0 .
$$

Let $t=\max \left\{t_{1}, \ldots, t_{n}\right\}$. Then

$$
\left(\mathfrak{c}^{t} H_{\mathfrak{b}}^{j}(A)\right)_{\mathfrak{p}_{i}}=0 \text { for all } i=1, \ldots, n,
$$

so that $\mathfrak{c}^{t} H_{\mathfrak{b}}^{j}(A)=0$ since $\operatorname{Ass}\left(\mathfrak{c}^{t} H_{\mathfrak{b}}^{j}(A)\right) \subseteq \operatorname{Ass}\left(H_{\mathfrak{b}}^{j}(A)\right)$.

\section{M-TORSION STRUCTURE}

We begin by extracting in a lemma the argument which forms the basis for the work in this section.

3.1 Lemma. Let $(A, \mathfrak{m})$ be a Gorenstein local ring of prime characteristic $p>$ 0 , and let $d: E^{\prime} \rightarrow E^{\prime \prime}$ be a homomorphism of injective $A$-modules such that $E^{\prime}$ is an essential extension of $\operatorname{Ker} d$. Suppose that each of $E^{\prime}$ and $E^{\prime \prime}$ has a direct summand isomorphic to $E:=E(A / \mathfrak{m})$, and let $q: E \rightarrow E^{\prime}$ and $\pi: E^{\prime \prime} \rightarrow E$ be the canonical injection and canonical projection (respectively) associated with these direct sum decompositions. By Matlis [M1, Theorems 3.6 and 3.7], E has a natural structure as a module over $\hat{A}$, the completion of $A$, and there exists a unique $\hat{a} \in \hat{A}$ such that the endomorphism $\pi \circ d \circ q$ of $E$ is given by multiplication by $\hat{a}$. 
Then $\hat{a} \in \widehat{\mathfrak{m}}$, the maximal ideal of $\hat{A} ;$ also, for each $e \in \mathbb{N}, F^{e}(E) \cong E(b y$ $1.4)$, and $F^{e}(\pi \circ d \circ q)$ is given by multiplication by $\hat{a}^{p^{e}}$.

Proof. Since $E^{\prime}$ is an essential extension of $\operatorname{Ker} d$, we have $\operatorname{Im} q \cap \operatorname{Ker} d \neq 0$, and so $\pi \circ d \circ q$ is not an automorphism of $E$. It follows that $\hat{a}$ is not a unit of $\hat{A}$, and so $\hat{a} \in \hat{\mathfrak{m}}$. It is straightforward to check the final claim.

3.2 Notation. Let $(A, \mathfrak{m})$ be a local ring. For an ideal $\mathfrak{b}$ of $A$, we shall denote by $\Gamma_{\mathfrak{b}}$ the $b$-torsion functor on the category of all $A$-modules: this is the subfunctor of the identity functor for which

$$
\Gamma_{\mathfrak{b}}(M)=\bigcup_{i=0}^{\infty}\left(0:_{M} \mathfrak{b}^{i}\right)
$$

for each $A$-module $M$. Of course, for $j \in \mathbb{N}_{0}$, the $j$ th local cohomology functor $H_{\mathfrak{b}}^{j}$ is just the $j$ th right derived functor of $\Gamma_{\mathfrak{b}}$.

3.3 Theorem. Let $(A, \mathfrak{m})$ be a regular local ring of characteristic $p>0$, and let $\mathfrak{b}$ be an ideal of $A$. Let $i, j \in \mathbb{N}_{0}$.

If $H_{\mathfrak{m}}^{i}\left(\operatorname{Ext}_{A}^{j}(A / \mathfrak{b}, A)\right)=0$, then $\mu^{i}\left(\mathfrak{m}, H_{\mathfrak{b}}^{j}(A)\right)=0$.

Proof. Let

$$
0 \longrightarrow \operatorname{Ext}_{A}^{j}(A / \mathfrak{b}, A) \longrightarrow E^{0} \longrightarrow \cdots \longrightarrow E^{i} \longrightarrow E^{i+1} \longrightarrow \cdots
$$

provide a minimal injective resolution for $\operatorname{Ext}_{A}^{j}(A / \mathfrak{b}, A)$. For each $i \in \mathbb{N}_{0}$, set $E_{0}^{i}=E^{i}, E_{1}^{i}=F\left(E^{i}\right)$, and, for each $n \in \mathbb{N}$, define $E_{n+1}^{i}=F\left(E_{n}^{i}\right)$.

The argument used in the proof of Theorem 2.1 shows that there is a commutative diagram

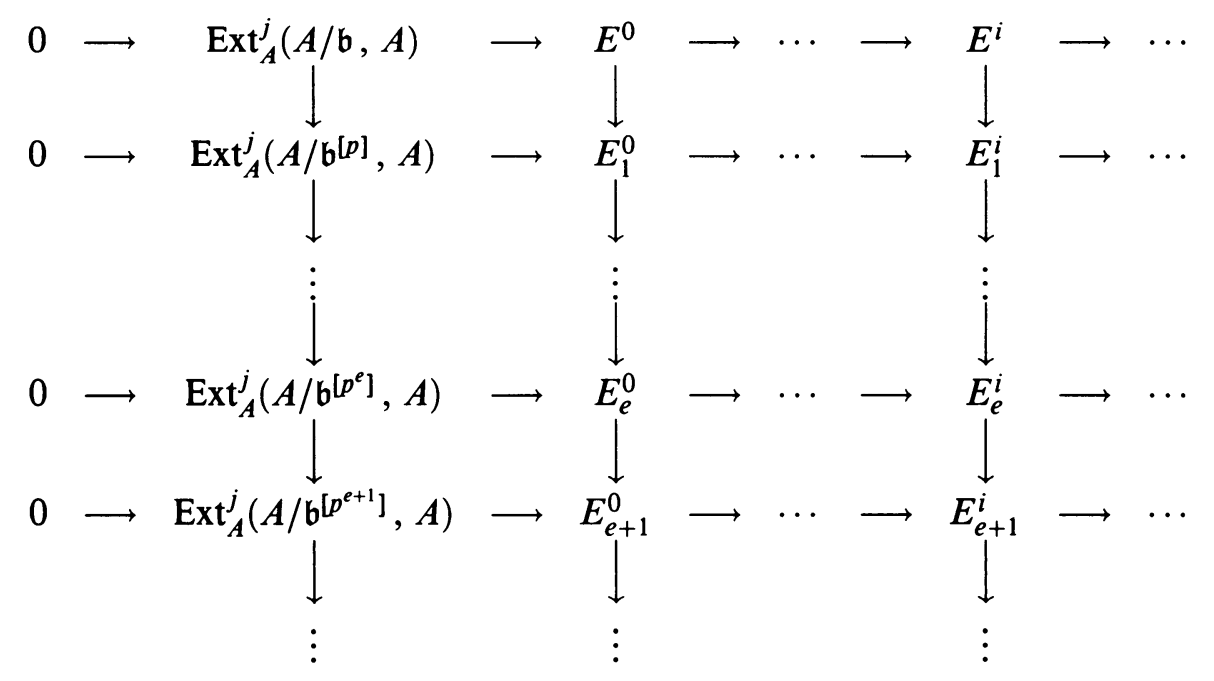

of $A$-modules and $A$-homomorphisms with exact rows, in which the maps in the first vertical column are all the natural ones, and the rows provide injective resolutions for the $\operatorname{Ext}_{A}^{j}\left(A / \mathfrak{b}^{\left[p^{e}\right]}, A\right)$; also, passage to direct limits produces an exact sequence

$$
0 \longrightarrow H_{\mathfrak{b}}^{j}(A) \longrightarrow J^{0} \longrightarrow \cdots \longrightarrow J^{i} \longrightarrow J^{i+1} \longrightarrow \cdots,
$$


where, for each $i \in \mathbb{N}_{0}$,

$$
J^{i}:=\underset{e \in \mathbb{N}}{\lim } E_{e}^{i}
$$

an injective $A$-module.

Since the Frobenius homomorphism $f: A \rightarrow A$ is flat, and the extension of $\mathfrak{m}$ to $A$ under $f$ (that is, the ideal $f(\mathfrak{m}) A^{\prime}$ of $A^{\prime}$ ) is just $\mathfrak{m}^{[p]}$, it follows from the theory of flat base change in local cohomology (and 1.3) that $H_{\mathrm{m}[p]}^{i}\left(\operatorname{Ext}_{A}^{j}\left(A / b^{[p]}, A\right)\right)=0$; hence

$$
H_{\mathfrak{m}}^{i}\left(\operatorname{Ext}_{A}^{j}\left(A / \mathfrak{b}^{\left[p^{e}\right]}, A\right)\right)=0 \text { for all } e \in \mathbb{N} .
$$

Thus the sequence $\Gamma_{\mathfrak{m}}\left(E_{e}^{i-1}\right) \rightarrow \Gamma_{\mathfrak{m}}\left(E_{e}^{i}\right) \rightarrow \Gamma_{\mathfrak{m}}\left(E_{e}^{i+1}\right)$ induced from the above commutative diagram is exact for all $e \in \mathbb{N}_{0}$. (Interpret $E_{e}^{-1}$ as 0 for all $e \in \mathbb{N}_{0}$.)

Let $x \in\left(0::_{J^{i}} \mathfrak{m}\right)$. Since $\mathfrak{m}$ is finitely generated, it follows that there exist $e \in \mathbb{N}$ and $y \in\left(0::_{E_{e}^{i}} \mathfrak{m}\right)$ such that its natural image in $J^{i}$ is $x$. By the comment in the immediately preceding paragraph, $y$ is the image of an element $z \in \Gamma_{\mathfrak{m}}\left(E_{e}^{i-1}\right)$. There exists $h \in \mathbb{N}$ such that $\mathfrak{m}^{\left[p^{h}\right]} z=0$. Hence, by 3.1 , the image of $y$ in $\left(0:_{E_{e+h}^{i}} \mathfrak{m}\right.$ ) (under the appropriate vertical map in the above commutative diagram) must be zero. (Go down and then along rather than along and then down!) Thus $x=0$.

We have therefore shown that $\left(0:_{J^{i}} \mathfrak{m}\right)=0$, so that $\operatorname{Ext}_{A}^{i}\left(A / \mathfrak{m}, H_{\mathfrak{b}}^{j}(A)\right)=0$ and $\mu^{i}\left(\mathfrak{m}, H_{\mathfrak{b}}^{j}(A)\right)=0$.

3.4 Corollary. Let $(A, \mathfrak{m})$ be a regular local ring of characteristic $p>0$, and let $\mathfrak{b}$ be an ideal of $A$. Let $j \in \mathbb{N}_{0}$ and let $\mathfrak{p} \in \operatorname{Spec}(A)$.

Then $\mu^{i}\left(\mathfrak{p}, H_{\mathfrak{b}}^{j}(A)\right)=0$ for all $i>\mathrm{ht} \mathfrak{p}-j$.

Proof. Localization enables us to reduce to the case in which $\mathfrak{p}=\mathfrak{m}$. Set $d:=\operatorname{dim} A$ and $E:=E(A / \mathfrak{m})$. By Grothendieck's local duality theorem [G1, 6.3],

$$
H_{\mathfrak{m}}^{i}\left(\operatorname{Ext}_{A}^{j}(A / \mathfrak{b}, A)\right) \cong \operatorname{Hom}_{A}\left(\operatorname{Ext}_{A}^{d-i}\left(\operatorname{Ext}_{A}^{j}(A / \mathfrak{b}, A), A\right), E\right) .
$$

However, since $A$ is Gorenstein, the latter module is zero whenever $d-i<j$, that is, whenever $i>d-j$ (by Bass [B, §1]). The claim therefore follows from 3.3.

3.5 Theorem. Let $(A, \mathfrak{m})$ be a regular local ring of characteristic $p>0$, and let $M$ be an A-module such that

(1) $F(M) \cong M$, and

(2) $\mu^{i}(\mathfrak{p}, M)$ is finite for all $i \in \mathbb{N}_{0}$ and all $\mathfrak{p} \in \operatorname{Spec}(A)$. Let

$$
0 \longrightarrow M \stackrel{\varepsilon}{\longrightarrow} E^{0} \longrightarrow \cdots \longrightarrow E^{i} \stackrel{d^{i}}{\longrightarrow} E^{i+1} \longrightarrow \cdots
$$

provide the minimal injective resolution for $M$.

Then, for all $i \in \mathbb{N}_{0}$, the restriction $\left.d^{i}\right|_{\Gamma_{\mathrm{m}}\left(E^{i}\right)}$ of $d^{i}$ to the submodule $\Gamma_{\mathrm{m}}\left(E^{i}\right)$ of $E^{i}$ is zero.

Consequently, for all $i \in \mathbb{N}_{0}$, the local cohomology module $H_{\mathfrak{m}}^{i}(M)$ is an injective A-module; in fact, $H_{\mathfrak{m}}^{i}(M)$ is isomorphic to the direct sum of $\mu^{i}(\mathfrak{m}, M)$ copies of $E(A / \mathfrak{m})$. In particular, $\Gamma_{\mathfrak{m}}(M)$ is an injective direct summand of $M$. 
Proof. Application of the exact functor $F$ yields an exact sequence

$$
0 \longrightarrow F(M) \stackrel{F(\varepsilon)}{\longrightarrow} F\left(E^{0}\right) \longrightarrow \cdots \longrightarrow F\left(E^{i}\right) \stackrel{F\left(d^{i}\right)}{\longrightarrow} F\left(E^{i+1}\right) \longrightarrow \cdots
$$

which, by 1.5 , provides an injective resolution for $F(M)$. Let $\theta^{-1}: M \rightarrow F(M)$ be the given isomorphism. By standard results about injective resolutions, there exist $A$-homomorphisms $\theta^{i}: E^{i} \rightarrow F\left(E^{i}\right)\left(i \in \mathbb{N}_{0}\right)$ such that the diagram

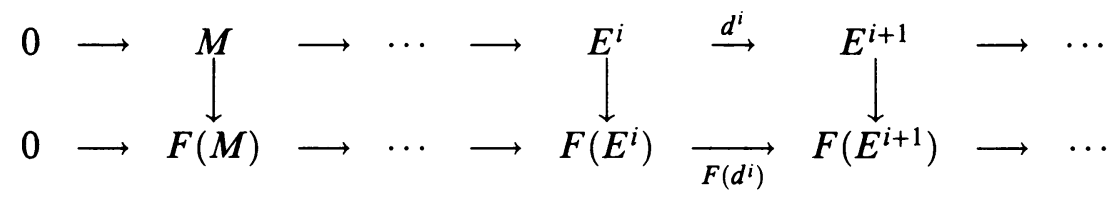

(in which the vertical maps are the $\theta^{i}(i \geqslant-1)$ ) commutes.

We can now use hypothesis (2) to deduce that all the $\theta^{i}\left(i \in \mathbb{N}_{0}\right)$ are isomorphisms, as follows. Suppose, inductively, that $i \in \mathbb{N}_{0}$ and we have proved that $\theta^{r}$ is an isomorphism for all $r=-1, \ldots, i-1$. Then a diagram-chase shows that $\operatorname{Ker} \theta^{i} \cap \operatorname{Ker} d^{i}=0$, and it follows that $\theta^{i}$ is a monomorphism because $E^{i}$ is an essential extension of $\operatorname{Ker} d^{i}$. Hence $\operatorname{Im} \theta^{i}$ is a direct summand of $F\left(E^{i}\right)$, and $\operatorname{Im} \theta^{i} \cong E^{i}$. Next, by $1.5, F\left(E^{i}\right) \cong E^{i}$, and so it follows from the fact that $\mu^{i}(\mathfrak{p}, M)$ is finite for all $\mathfrak{p} \in \operatorname{Spec}(A)$, together with the uniqueness properties of the decomposition of $E^{i}$ as a direct sum of indecomposable injective submodules, that $\operatorname{Im} \theta^{i}=F\left(E^{i}\right)$. Hence $\theta^{i}$ is an isomorphism, and the inductive step is complete.

For each $i \in \mathbb{N}_{0}$, set $d_{1}^{i}=F\left(d^{i}\right), E_{1}^{i}=F\left(E^{i}\right)$, and, for each $n \in \mathbb{N}$, define $E_{n+1}^{i}=F\left(E_{n}^{i}\right), d_{n+1}^{i}=F\left(d_{n}^{i}\right)$. Repeated application of the functor $F$ to the last commutative diagram therefore yields a commutative diagram

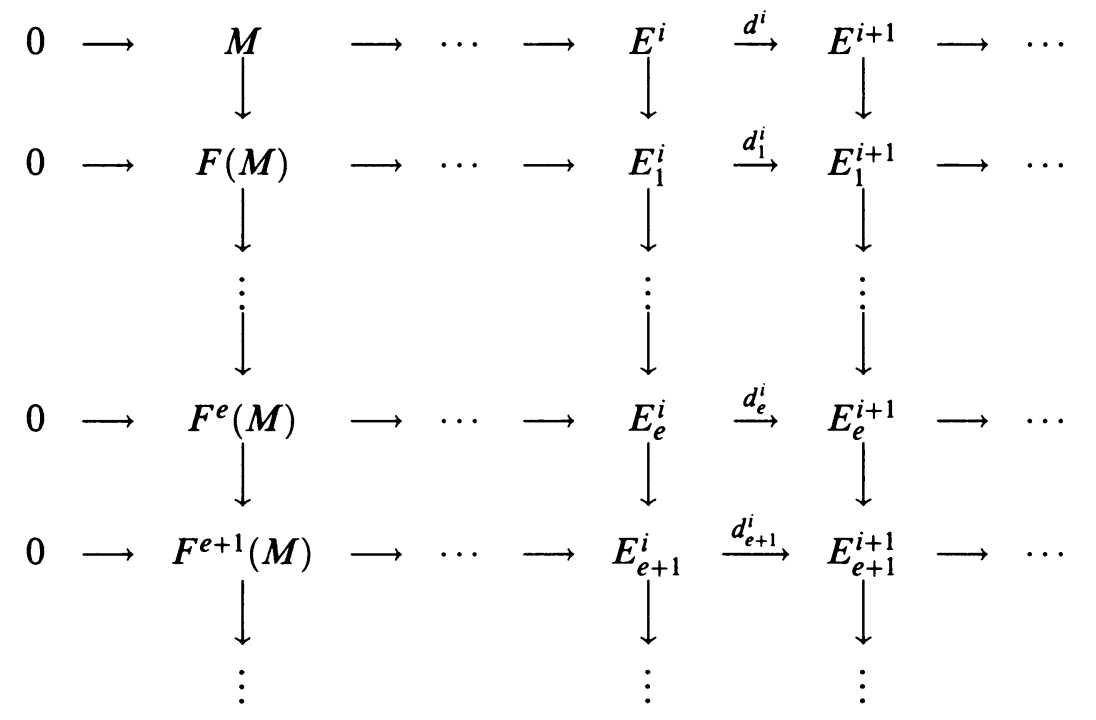

in which the vertical maps are all isomorphisms and the rows are all exact.

Now let $x \in \Gamma_{\mathfrak{m}}\left(E^{i}\right)$. There exists $e \in \mathbb{N}$ such that $\mathrm{m}^{\left[p^{e}\right]} x=0$. It therefore follows from 3.1 that

$$
F^{e-1}\left(\theta^{i+1}\right) \cdots F\left(\theta^{i+1}\right) \theta^{i+1} d^{i}(x)=F^{e}\left(d^{i}\right) F^{e-1}\left(\theta^{i}\right) \cdots F\left(\theta^{i}\right) \theta^{i}(x)=0,
$$

so that $d^{i}(x)=0$ as $\theta^{i+1}$ is an isomorphism. 
The remaining claims of the theorem are now immediate.

3.6 Corollary. Let $(A, \mathfrak{m})$ be a regular local ring of characteristic $p>0$, and let $M$ be an Artinian $A$-module such that $F(M) \cong M$. Then $M$ is an injective A-module.

Proof. It follows from Matlis [M2, Proposition 3], together with the fact that $M$ is Artinian, that $\mu^{i}(\mathfrak{p}, M)=0$ for all $i \in \mathbb{N}_{0}$ and all $\mathfrak{p} \in \operatorname{Spec}(A) \backslash\{\mathfrak{m}\}$, and that $\mu^{i}(\mathfrak{m}, M)$ is finite for all $i \in \mathbb{N}_{0}$.

We can therefore apply Theorem 3.5 to deduce that $\Gamma_{\mathfrak{m}}(M)$ is injective, and since $M=\Gamma_{\mathfrak{m}}(M)$, the result is proved.

3.7 Corollary. Let $(A, \mathfrak{m})$ be a regular local ring of characteristic $p>0$, and let $\mathfrak{b}$ be an ideal of $A$. Let $i, j \in \mathbb{N}_{0}$.

Then $H_{\mathfrak{m}}^{i}\left(H_{\mathfrak{b}}^{j}(A)\right)$ is an injective A-module; in fact $H_{\mathfrak{m}}^{i}\left(H_{\mathfrak{b}}^{j}(A)\right)$ is isomorphic to the direct sum of $\mu^{i}\left(\mathfrak{m}, H_{\mathfrak{b}}^{j}(A)\right)$ copies of $E(A / \mathfrak{m}) \quad\left(\right.$ and $\mu^{i}\left(\mathfrak{m}, H_{\mathfrak{b}}^{j}(A)\right)$ is finite, by 2.1).

In particular, $\Gamma_{\mathfrak{m}}\left(H_{\mathfrak{b}}^{j}(A)\right)$ is an injective direct summand of $H_{\mathfrak{b}}^{j}(A)$.

Proof. By 1.8, $F\left(H_{\mathfrak{b}}^{j}(A)\right) \cong H_{\mathfrak{b}}^{j}(A)$. Also, by $2.1, \mu^{r}\left(\mathfrak{p}, H_{\mathfrak{b}}^{j}(A)\right)$ is finite for all $r \in \mathbb{N}_{0}$ and all $\mathfrak{p} \in \operatorname{Spec}(A)$. The result therefore follows from 3.5.

3.8 Corollary. (Compare Hartshorne and Speiser [H-S, 2.4].) Let $(A, \mathfrak{m})$ be a regular local ring of characteristic $p>0$, and let $\mathfrak{b}$ be an ideal of $A$. Let $j \in \mathbb{N}_{0}$.

If $H_{\mathfrak{b}}^{j}(A)$ is Artinian, then it is injective and isomorphic to a direct sum of finitely many copies of $E_{A}(A / \mathfrak{m})$.

Proof. By 1.8, $F\left(H_{\mathfrak{b}}^{j}(A)\right) \cong H_{\mathfrak{b}}^{j}(A)$. The result therefore follows from 3.6.

Before we state our last corollary in this section, we remind the reader that, for a nonzero module $M$ over a local ring $A$, the dimension of $M$, denoted by $\operatorname{dim} M$ or $\operatorname{dim}_{A} M$, is the supremum of lengths of chains of prime ideals in $\operatorname{Supp} M$.

The next corollary is at least as good as Corollary 3.4 , because a localization argument shows, in the notation of 3.4 , that $\operatorname{dim} H_{\mathfrak{b}}^{j}(A) \leq \operatorname{dim} A-j$ when $H_{\mathfrak{b}}^{j}(A) \neq 0$ (we are grateful to G. Lyubeznik for drawing our attention to this).

3.9 Corollary. Let $(A, \mathfrak{m})$ be a regular local ring of characteristic $p>0$, and let $\mathfrak{b}$ be an ideal of $A$. Let $j \in \mathbb{N}_{0}$ and let $\mathfrak{p} \in \operatorname{Supp}\left(H_{\mathfrak{b}}^{j}(A)\right)$.

Then $\mu^{i}\left(\mathfrak{p}, H_{\mathfrak{b}}^{j}(A)\right)=0$ for all $i>\operatorname{dim}_{A_{\mathfrak{p}}} H_{\mathfrak{b} A_{\mathfrak{p}}}^{j}\left(A_{\mathfrak{p}}\right)=\operatorname{dim}_{A_{\mathfrak{p}}}\left(H_{\mathfrak{b}}^{j}(A)\right)_{\mathfrak{p}}$.

Proof. Of course, $H_{\mathfrak{b} A_{\mathfrak{p}}}^{j}\left(A_{\mathfrak{p}}\right) \cong\left(H_{\mathfrak{b}}^{j}(A)\right)_{\mathfrak{p}}$ (as $A_{\mathfrak{p}}$-modules). We argue by induction on $n:=\operatorname{dim}_{A_{\mathfrak{p}}}\left(H_{\mathfrak{b}}^{j}(A)\right)_{\mathfrak{p}}$.

When $n=0$, we have $\operatorname{Supp}_{A_{\mathfrak{p}}}\left(\left(H_{\mathfrak{b}}^{j}(A)\right)_{\mathfrak{p}}\right)=\left\{\mathfrak{p} A_{\mathfrak{p}}\right\}$. It therefore follows from Matlis [M2, Proposition 3], together with the fact (2.1) that $\mu^{0}\left(\mathfrak{p}, H_{\mathfrak{b}}^{j}(A)\right)$ is finite, that $H_{\mathfrak{b} A_{\mathfrak{p}}}^{j}\left(A_{\mathfrak{p}}\right)$ is an Artinian $A_{\mathfrak{p}}$-module. Since $A_{\mathfrak{p}}$ is a regular local ring of characteristic $p$, it follows from 3.8 that $H_{\mathfrak{b} A_{\mathfrak{p}}}^{j}\left(A_{\mathfrak{p}}\right)$ is injective. Hence $\mu^{i}\left(\mathfrak{p}, H_{\mathfrak{b}}^{j}(A)\right)=0$ for all $i>0$. 
Now suppose, inductively, that $n=k>0$, and that the result has been proved for all smaller values of $n$. Let

$$
0 \longrightarrow H_{\mathfrak{b}}^{j}(A) \stackrel{\varepsilon}{\longrightarrow} E^{0} \longrightarrow \cdots \longrightarrow E^{i} \stackrel{d^{i}}{\longrightarrow} E^{i+1} \longrightarrow \cdots
$$

provide a minimal injective resolution for $H_{\mathfrak{b}}^{j}(A)$. It follows from 2.1 and the inductive hypothesis that, for all $i \geqslant k$, the injective $A_{\mathfrak{p}}$-module $\left(E^{i}\right)_{\mathfrak{p}}$ is isomorphic to a direct sum of finitely many copies of $E_{A_{\mathfrak{p}}}\left(A_{\mathfrak{p}} / \mathfrak{p} A_{\mathfrak{p}}\right)$. But

$$
0 \longrightarrow\left(H_{\mathfrak{b}}^{j}(A)\right)_{\mathfrak{p}} \stackrel{(\varepsilon)_{\mathfrak{p}}}{\longrightarrow}\left(E^{0}\right)_{\mathfrak{p}} \longrightarrow \cdots \longrightarrow\left(E^{i}\right)_{\mathfrak{p}} \stackrel{\left(d^{i}\right)_{\mathfrak{p}}}{\longrightarrow}\left(E^{i+1}\right)_{\mathfrak{p}} \longrightarrow \cdots
$$

provides a minimal injective resolution for $\left(H_{\mathfrak{b}}^{j}(A)\right)_{\mathfrak{p}} \cong H_{\mathfrak{b} A_{\mathfrak{p}}}^{j}\left(A_{\mathfrak{p}}\right)$, and so it follows from 1.8, 2.1 and 3.5 that $\left(d^{i}\right)_{\mathfrak{p}}=0$ for all $i \geqslant k$, so that $\left(E^{i}\right)_{\mathfrak{p}}=0$ for all $i>k$. This implies that $\mu^{i}\left(\mathfrak{p}, H_{\mathfrak{b}}^{j}(A)\right)=0$ for all $i>k$, and so the inductive step is complete.

\section{SOME QUESTIONS}

In this section, we state some questions about regular local rings of characteristic 0 which are raised by our results in $\S \S 2$ and 3 above, and assemble some low-dimensional and other evidence related to them. Our first question is motivated by Theorem 2.1 .

4.1 Question. Let $(A, \mathfrak{m})$ be a regular local ring of characteristic 0 , and let $\mathfrak{b}$ be an ideal of $A$. Let $\mathfrak{p} \in \operatorname{Spec}(A)$, and let $j \in \mathbb{N}_{0}$.

Is it the case that $\mu^{i}\left(\mathfrak{p}, H_{\mathfrak{b}}^{j}(A)\right)$ is finite for all $i \in \mathbb{N}_{0}$ ?

4.2 Notation and remarks. Let $\mathscr{I}(A)$ denote the set of all ideals of $A$, and let

$$
\mathscr{G}(A):=\left\{\mathfrak{b} \in \mathscr{I}(A): \mu^{i}\left(\mathfrak{p}, H_{\mathfrak{b}}^{j}(A)\right) \text { is finite for all } i, j \in \mathbb{N}_{0}\right.
$$

$$
\text { and all } \mathfrak{p} \in \operatorname{Spec}(A)\} \text {. }
$$

Trivially, $0 \in \mathscr{G}(A)$.

Let $\mathfrak{b}, \mathfrak{c}$ be ideals of $A$. Observe that $\mathfrak{b} \in \mathscr{G}(A)$ if and only if $\sqrt{ } \mathfrak{b} \in \mathscr{G}(A)$; therefore $\mathfrak{b} \cap \mathfrak{c} \in \mathscr{G}(A)$ if and only if $\mathfrak{b} \mathfrak{c} \in \mathscr{G}(A)$.

Note that for a local ring $(A, \mathfrak{m})$, it is automatic that $\mathfrak{m} \in \mathscr{G}(A)$, since $H_{\mathfrak{m}}^{j}(A)$ is Artinian for all $j \in \mathbb{N}_{0}$.

4.3 Lemma. Let $a \in A$. Let $\mathfrak{p} \in \operatorname{Spec}(A)$, and let $j \in \mathbb{N}_{0}$.

Then $\mu^{i}\left(\mathfrak{p}, H_{A a}^{j}(A)\right)$ is finite for all $i \in \mathbb{N}_{0}$.

Proof. Since $A a$ is principal, $H_{A a}^{j}(A)=0$ for all $j>1$; also $H_{A a}^{0}(A)$ is finitely generated, and so $\mu^{i}\left(\mathfrak{p}, H_{A a}^{0}(A)\right)$ is finite for all $i \in \mathbb{N}_{0}$.

There is an integer $h$ such that $\left(0:_{A} a^{h}\right)=\left(0:_{A} a^{h+i}\right)$ for all $i \in \mathbb{N}$; furthermore, the Cech complex approach to the calculation of local cohomology modules shows that there is an exact sequence

$$
0 \longrightarrow A /\left(0:_{A} a^{h}\right) \longrightarrow A_{a} \longrightarrow H_{A a}^{1}(A) \longrightarrow 0 .
$$

The result follows from this exact sequence: of course, $\mu^{i}\left(\mathfrak{p}, A /\left(0:_{A} a^{h}\right)\right)$ is finite for all $i \in \mathbb{N}_{0}$, since $A /\left(0:_{A} a^{h}\right)$ is finitely generated; moreover $\mu^{i}\left(\mathfrak{p}, A_{a}\right)$ 
is finite for all $i \in \mathbb{N}_{0}$, since a minimal injective resolution for $A_{a}$ as $A_{a^{-}}$ module is automatically an injective resolution for $A_{a}$ as $A$-module, and, for a prime ideal $\mathfrak{q}$ of $A$ which does not contain $a$, the indecomposable injective $A_{a}$-module $E_{A_{a}}\left(A_{a} / \mathfrak{q} A_{a}\right)$ is, when considered as an $A$-module, isomorphic to $E_{A}(A / \mathfrak{q})$.

4.4 Lemma. Let $(A, \mathfrak{m})$ be a regular local ring of characteristic 0 and let $\mathfrak{b}$ be a proper ideal of $A$ such that $\operatorname{dim} A / \mathfrak{b}=1$. Let $\mathfrak{p} \in \operatorname{Spec}(A)$, and let $j \in \mathbb{N}_{0}$.

Then $\mu^{i}\left(\mathfrak{p}, H_{\mathfrak{b}}^{j}(A)\right)$ is finite for all $i \in \mathbb{N}_{0}$.

Proof. Since $H_{\mathrm{b}}^{0}(A)$ is finitely generated, the result is clear when $j=0$, and so we can assume henceforth in this proof that $j \geqslant 1$. Also we can, and do, assume that $\mathfrak{p} \supseteq \mathfrak{b}$, since otherwise $\mathfrak{p} \notin \operatorname{Supp}\left(H_{\mathfrak{b}}^{j}(A)\right)$.

Let $i \in \mathbb{N}_{0}$. By [H-K, 4.1], $\operatorname{Ext}_{\hat{A}}^{i}\left(\hat{A} / \mathfrak{p} \hat{A}, H_{\mathfrak{b}}^{j}(A) \otimes_{A} \hat{A}\right)$ is a finitely generated $\hat{A}$-module, so that, since the natural ring homomorphism $A \rightarrow \hat{A}$ is faithfully flat, $\operatorname{Ext}_{A}^{i}\left(A / \mathfrak{p}, H_{\mathfrak{b}}^{j}(A)\right)$ is a finitely generated $A$-module. Hence $\mu^{i}\left(\mathfrak{p}, H_{\mathfrak{b}}^{j}(A)\right)$ is finite.

4.5 Proposition. Let $(A, \mathfrak{m})$ be a regular local ring of characteristic 0 having $\operatorname{dim} A \leqslant 3$, and let $\mathfrak{b}$ be an ideal of $A$. Let $\mathfrak{p} \in \operatorname{Spec}(A)$, and let $j \in \mathbb{N}_{0}$.

Then $\mu^{i}\left(\mathfrak{p}, H_{\mathfrak{b}}^{j}(A)\right)$ is finite for all $i \in \mathbb{N}_{0}$.

Proof. Since $A$ is a unique factorization domain, an unmixed proper radical ideal of $A$ of height 1 is principal, and so we can use 4.2, 4.3 and 4.4 to reduce the proof to the case where $\operatorname{dim} A=3$ and $\mathfrak{b}$ is a proper, mixed, radical ideal of $A$ of height 1 .

In this situation, there exist a nonunit, nonzero divisor $a \in A$ and an unmixed ideal $\mathfrak{c}$ of $A$ of height 2 such that $\mathfrak{b}=A a \cap \mathfrak{c}$ and $\sqrt{ }(A a+\mathfrak{c})=\mathfrak{m}$. We use the notation $\mathscr{G}(A)$ of 4.2. By 4.2, $\mathrm{m} \in \mathscr{G}(A)$; by $4.3, A a \in \mathscr{G}(A)$; and, by 4.4, c $\in \mathscr{G}(A)$. By the Mayer-Vietoris sequence for local cohomology, there is an exact sequence

$$
\begin{aligned}
& 0 \quad \longrightarrow \quad H_{\mathfrak{m}}^{0}(A) \quad \longrightarrow \quad H_{A a}^{0}(A) \oplus H_{\mathfrak{c}}^{0}(A) \longrightarrow H_{\mathfrak{b}}^{0}(A) \\
& \longrightarrow H_{\mathfrak{m}}^{1}(A) \longrightarrow H_{A a}^{1}(A) \oplus H_{\mathfrak{c}}^{1}(A) \longrightarrow H_{\mathfrak{b}}^{1}(A) \\
& \longrightarrow H_{\mathrm{m}}^{2}(A) \longrightarrow H_{A a}^{2}(A) \oplus H_{\mathfrak{c}}^{2}(A) \longrightarrow H_{\mathfrak{b}}^{2}(A) \\
& \longrightarrow H_{\mathrm{m}}^{3}(A) \quad \longrightarrow H_{A a}^{3}(A) \oplus H_{\mathrm{c}}^{3}(A) \quad \longrightarrow \quad H_{\mathrm{b}}^{3}(A) \longrightarrow 0 \text {. }
\end{aligned}
$$

Now $H_{A a}^{j}(A) \neq 0$ if and only if $j=1$; furthermore, since grade $\mathfrak{c}=2$, we have $H_{\mathrm{c}}^{j}(A)=0$ if $j=0$ or 1 . Also, by the local Lichtenbaum-Hartshorne Theorem [H1, 3.1], $H_{\mathrm{c}}^{3}(A)=H_{\mathrm{b}}^{3}(A)=0$. Thus the above exact sequence shows that $H_{\mathfrak{b}}^{0}(A)=0$, that $H_{\mathfrak{b}}^{1}(A) \cong H_{A a}^{1}(A)$, and that there is an exact sequence

$$
0 \longrightarrow H_{\mathfrak{c}}^{2}(A) \longrightarrow H_{\mathfrak{b}}^{2}(A) \longrightarrow H_{\mathrm{m}}^{3}(A) \longrightarrow 0 \text {. }
$$

Since $\mathfrak{m}, \mathfrak{c}, A a \in \mathscr{G}(A)$, it follows that $\mathfrak{b} \in \mathscr{G}(A)$ too, as required.

4.6 Proposition. Let $(A, \mathfrak{m})$ be a 4-dimensional regular local ring containing a field of characteristic 0 , and let $\mathfrak{b}$ be an ideal of $A$. Let $\mathfrak{p} \in \operatorname{Spec}(A)$, and let $j \in \mathbb{N}_{0}$.

Then $\mu^{i}\left(\mathfrak{p}, H_{\mathfrak{b}}^{j}(A)\right)$ is finite for all $i \in \mathbb{N}_{0}$.

Proof. We first consider a proper, unmixed ideal $\hat{\mathfrak{b}}$ of height 2 of the completion $(\hat{A}, \hat{\mathfrak{m}})$. Since grade $\hat{\mathfrak{b}}=2$, we have $H_{\hat{\mathfrak{b}}}^{j}(\hat{A})=0$ if $j=0$ or 1 . Also, 
by the local Lichtenbaum-Hartshorne Theorem $[\mathrm{H} 1,3.1], H_{\hat{b}}^{4}(\hat{A})=0$. Let the minimal injective resolution for $\hat{A}$ (as a module over itself) be

$$
0 \longrightarrow \hat{A} \longrightarrow E^{0} \longrightarrow E^{1} \longrightarrow E^{2} \longrightarrow E^{3} \longrightarrow E^{4} \longrightarrow 0 .
$$

Two cases now arise, according as $\operatorname{Spec}(\hat{A} / \hat{\mathfrak{b}}) \backslash\{\hat{\mathfrak{m}} / \hat{\mathfrak{b}}\}$ is or is not connected.

When $\operatorname{Spec}(\hat{A} / \hat{\mathfrak{b}}) \backslash\{\hat{\mathfrak{m}} / \hat{\mathfrak{b}}\}$ is connected, it follows from [H-L, 2.9] that $H_{\hat{\mathfrak{b}}}^{3}(\hat{A})$ $=0$ also; hence, since $\Gamma_{\hat{b}}\left(E^{0}\right)=\Gamma_{\hat{b}}\left(E^{1}\right)=0$, there is induced an exact sequence

$$
0 \longrightarrow H_{\hat{\mathfrak{b}}}^{2}(\hat{A}) \longrightarrow \Gamma_{\hat{\mathfrak{b}}}\left(E^{2}\right) \longrightarrow \Gamma_{\hat{\mathfrak{b}}}\left(E^{3}\right) \longrightarrow \Gamma_{\hat{\mathfrak{b}}}\left(E^{4}\right) \longrightarrow 0,
$$

and, since this provides an injective resolution for $H_{\hat{b}}^{2}(\hat{A})$, it is now obvious that $\hat{\mathfrak{b}} \in \mathscr{G}(\hat{A})$ (in the notation of 4.2).

When $\operatorname{Spec}(\hat{A} / \hat{\mathfrak{b}}) \backslash\{\hat{\mathfrak{m}} / \hat{\mathfrak{b}}\}$ has $t>1$ distinct connected components, it follows from $[\mathrm{H}-\mathrm{K}, 3.6]$ that $H_{\hat{\mathfrak{b}}}^{3}(\hat{A}) \cong E_{\hat{A}}(\hat{A} / \hat{\mathfrak{m}})^{t-1}$, the direct sum of $t-1$ copies of $E_{\hat{A}}(\hat{A} / \hat{\mathfrak{m}})$. Thus in this case there exist $\hat{A}$-modules $K$ and $I$ and exact sequences

$$
\begin{gathered}
0 \longrightarrow K \longrightarrow \Gamma_{\hat{\mathfrak{b}}}\left(E^{3}\right) \longrightarrow \Gamma_{\hat{\mathfrak{b}}}\left(E^{4}\right) \longrightarrow 0 \\
0 \longrightarrow I \longrightarrow K \longrightarrow E_{\hat{A}}(\hat{A} / \hat{\mathfrak{m}})^{t-1} \longrightarrow 0
\end{gathered}
$$

and

$$
0 \longrightarrow H_{\hat{\mathfrak{b}}}^{2}(\hat{A}) \longrightarrow \Gamma_{\hat{\mathfrak{b}}}\left(E^{2}\right) \longrightarrow I \longrightarrow 0,
$$

and it follows easily from these that $\hat{\mathfrak{b}} \in \mathscr{G}(\hat{A})$ in this case too.

Next suppose that $\hat{\mathfrak{b}}$ is a proper, mixed, radical ideal of $\hat{A}$ of height 2 , so that there exist proper, unmixed, radical ideals $\hat{\mathfrak{c}}$ and $\hat{\mathfrak{d}}$ of $\hat{A}$ with $\mathrm{ht} \hat{\mathfrak{c}}=2$, ht $\hat{\mathfrak{d}}=3, \sqrt{ }(\hat{\mathfrak{c}}+\hat{\mathfrak{d}})=\widehat{\mathfrak{m}}$ and $\hat{\mathfrak{c}} \cap \hat{\mathfrak{d}}=\hat{\mathfrak{b}}$. By the first three paragraphs of this proof, $\hat{\mathfrak{c}} \in \mathscr{G}(\hat{A})$; by $4.4, \hat{\mathfrak{d}} \in \mathscr{G}(\hat{A})$ too. It now follows from an argument using a Mayer-Vietoris sequence very similar to that used in the proof of 4.5 that $\hat{\mathfrak{b}} \in \mathscr{G}(\hat{A})$ too.

Now let us consider the ideal $\mathfrak{b}$ of $A$. We can assume that $\mathfrak{b} \subseteq \mathfrak{m}$. We consider first the case in which ht $\mathfrak{b}=2$. Localization and 4.5 enable us to see that $\mu^{i}\left(\mathfrak{p}, H_{\mathfrak{b}}^{j}(A)\right)$ is finite for all $i, j \in \mathbb{N}_{0}$ and all $\mathfrak{p} \in \operatorname{Spec}(A) \backslash\{\mathfrak{m}\}$. By (4.2 and) the first four paragraphs of this proof, $\mu^{i}\left(\widehat{\mathfrak{m}}, H_{\mathfrak{b} \hat{A}}^{j}(\hat{A})\right)$ is finite for all $i, j \in \mathbb{N}_{0}$, and it follows that $\mu^{i}\left(\mathfrak{m}, H_{\mathfrak{b}}^{j}(A)\right)$ is finite for all $i, j \in \mathbb{N}_{0}$. Thus $\mathfrak{b} \in \mathscr{G}(A)$ in this case.

We can now use $4.2,4.3$ and 4.4 to reduce the proof to the case where $\mathfrak{b}$ is a proper, mixed, radical ideal of $A$ of height 1 . In this situation, there exist a nonunit, nonzero divisor $a \in A$ and a proper ideal $\mathrm{c}$ of $A$ of height at least 2 such that $\mathfrak{b}=A a \cap \mathfrak{c}$ and $\operatorname{ht}(A a+\mathfrak{c}) \geqslant 3$. The arguments above show that $\mathfrak{c} \in$ $\mathscr{G}(A)$; in order to show that $\mathfrak{b} \in \mathscr{G}(A)$ in this case, it is enough, by 4.2 , to show that $a \mathfrak{c} \in \mathscr{G}(A)$. However, it is not difficult to use the Čech complex approach to the calculation of local cohomology modules to see that $H_{a c}^{j}(A) \cong\left(H_{c}^{j}(A)\right)_{a}$ for all $j \geqslant 2$. We already know that $\mu^{i}\left(\mathfrak{p}, H_{\mathfrak{c}}^{j}(A)\right)$ is finite for all $i, j \in \mathbb{N}_{0}$ and all $\mathfrak{p} \in \operatorname{Spec}(A)$. It therefore follows from standard considerations about the behaviour of injective modules under fraction formation that $\mu^{i}\left(\mathfrak{p}, H_{a c}^{j}(A)\right)$ is finite for all $i, j \in \mathbb{N}_{0}$ with $j \geqslant 2$ and all $\mathfrak{p} \in \operatorname{Spec}(A)$. Finally, $H_{a c}^{0}(A)=0$ and $H_{a c}^{1}(A) \cong H_{a A}^{1}(A)$ by another Mayer-Vietoris sequence argument, and so another application of 4.3 completes the proof. 
4.7 Question. Let $(A, \mathfrak{m})$ be a regular local ring of characteristic 0 , and let $\mathfrak{b}$ be an ideal of $A$. Let $j \in \mathbb{N}_{0}$.

If $H_{\mathfrak{b}}^{j}(A)$ is Artinian, must it be injective?

4.8 Remarks. Of course, Question 4.7 is motivated by 3.8. As further related evidence, we point out that, by [H-K, 3.6], if $(A, \mathfrak{m})$ is a complete regular local ring of dimension $d$ which contains a field of characteristic 0 and is such that $\operatorname{Spec}(A / \mathfrak{b}) \backslash\{\mathfrak{m} / \mathfrak{b}\}$ is not connected, where $\mathfrak{b}$ is a proper, unmixed ideal of $A$ having $\operatorname{dim}(A / \mathfrak{b})=2$, then $H_{\mathfrak{b}}^{d-1}(A)$ is Artinian and injective.

\section{REFERENCES}

[B] H. Bass, On the ubiquity of Gorenstein rings, Math. Z. 82 (1963), 8-28.

[G1] A. Grothendieck, Local cohomology, Lecture Notes in Math., vol. 41, Springer, Berlin, 1967.

[G2] Cohomologie locale des faisceaux coherents at théorèmes de Lefschetz locaux et globaux (SGA 2), North-Holland, Amsterdam, 1968.

[H1] R. Hartshorne, Cohomological dimension of algebraic varieties, Ann. of Math. 88 (1968), 403-450.

[H2] - Affine duality and cofiniteness, Invent. Math. 9 (1970), 145-164.

[H-S] R. Hartshorne and R. Speiser, Local cohomological dimension in characteristic p, Ann. of Math. 105 (1977), 45-79.

[H-K] C. Huneke and J. Koh, Cofiniteness and vanishing of local cohomology modules, Math. Proc. Cambridge Philos. Soc. 110 (1991), 421-429.

[H-L] C. Huneke and G. Lyubeznik, On the vanishing of local cohomology modules, Invent. Math. 102 (1990), 73-93.

[K] E. Kunz, Characterizations of regular local rings of characteristic p, Amer. J. Math. 91 (1969), 772-784.

[M1] E. Matlis, Injective modules over noetherian rings, Pacific J. Math. 8 (1958), 511-528.

[M2] _ Modules with descending chain condition, Trans. Amer. Math. Soc. 97 (1960), 495508.

[P-S] C. Peskine and L. Szpiro, Dimension projective finie et cohomologie locale, Inst. Hautes Études Sci. Publ. Math. 42 (1973), 323-395.

Department of Mathematics, Purdue University, West Lafayette, Indiana 47907

E-mail address: huneke@math.purdue.edu

Department of Pure Mathematics, University of Sheffield, Hicks Building, Sheffield S3 7RH, UK

E-mail address: r.y.sharp@sheffield.ac.uk 